\title{
Erythrocytic antioxidant system in the administration of new coordination compounds thiosemicarbazide derivatives
}

\author{
${ }^{\star 1}$ Valeriana Pantea, ${ }^{1}$ Marina Gamaniuc, ${ }^{2}$ Veaceslav Popa \\ ${ }^{1}$ Laboratory of Biochemistry, ${ }^{2}$ Department of Biochemistry and Clinical Biochemistry \\ Nicolae Testemitanu State University of Medicine and Pharmacy, Chisinau, the Republic of Moldova \\ Authors' ORCID iDs, academic degrees and contribution are available at the end of the article \\ *Corresponding author - Valeriana Pantea, e-mail: valeriana.pantea@usmf.md \\ Manuscript received October 15, 2021; revised manuscript November 18, 2021; published online November 26, 2021
}

\begin{abstract}
Background: Currently, there is a growing interest in new local coordination compounds (CC), which demonstrated antitumor properties, but their influence on the erythrocyte antioxidant system has not been studied. The aim of the study: to study the effects of CC, thiosemicarbazide derivatives -TIA-160, CMT-67 and CMJ-33 on indices of the antioxidant system indicators in erythrocytes peripheral blood in vivo experiments.

Material and methods: The action of CC on superoxide dismutase, catalase, total antioxidant capacity was evaluated on a group of 34 white rats, randomly divided into 4 groups: the first control group was injected subcutaneously with saline. The other groups (2 - TIA-160, 3 - CMT-67 and 4 - CMJ-33) were given subcutaneously 3 times a week for 30 days, $0.1 \mu \mathrm{M} / \mathrm{kg} \mathrm{CC}$.

Results: It was established that the TIA-160 compound demonstrated the highest capacity to induce the expression of erythrocyte catalase that exceeded the control level of 1.8, which did not correlate with the enzymatic superoxide dismutase (SOD) activity. Thus, this study showed that there are differences in the mechanisms of action of thiosemicarbazone derivatives.

Conclusions: The influence of tested CC on the indices of the antioxidant system is selective. This selectivity may be the base to their strong selective antiproliferative and cytotoxic action on tumor cells, but not on healthy ones.
\end{abstract}

Key words: coordination compounds, erythrocytes, antioxidant enzymes.

\section{Cite this article}

Pantea V, Gamaniuc M, Popa V. Eritrocytic antioxidant system in the administration of new coordination compounds thiosemicarbazide derivatives Mold Med J. 2021;64(5):70-75. https://doi.org/10.52418/moldovan-med-j.64-5.21.13.

\section{Introduction}

Extensive research over the past two decades has revealed the mechanism by which continuous oxidative stress can lead to chronic inflammation, which can lead to a lot of chronic diseases, including cancer, diabetes, cardiovascular, neurological and lung diseases. Oxidative stress can activate a variety of transcription factors. Activation of these transcription factors can lead to the expression of over 500 different genes, including those for growth factors, inflammatory cytokines, chemokines, cell cycle regulatory molecules and anti-inflammatory molecules. The way that oxidative stress activates the inflammatory pathways leads to the transformation of a normal cell into tumor cells, tumor cell survival, and proliferation, chemoresistance, radioresistance, invasion, angiogenesis, and stem cell survival is the focus of this review. In general, observations to date suggest that oxidative stress, chronic inflammation and cancer are closely linked. The generation of reactive oxygen radicals in mammalian cells profoundly affects many critical cellular functions, and the absence of effective cellular detoxification mechanisms that eliminate these radicals can lead to more human diseases.

Growing evidence suggests that reactive oxygen species
(ROS) in cells act as the second messenger in intracellular signaling cascades that induce and maintain the oncogenic phenotype of cancer cells. ROS are tumorigenic due to their ability to increase cell proliferation, survival, cell migration and also by inducing deoxyribonucleic acid (DNA) damage, leading to genetic lesions that initiate tumorigenicity and support subsequent tumor progression. However, it is also known that ROS can induce cell senescence and cell death and therefore function as antitumorigenic agents. Therefore, the mechanisms by which cells respond to reactive oxygen species depend on the molecular background of cells and tissues, the location of ROS production, and the concentration of individual ROS species. Carcinoma cells produce ROS at high rates in vitro and in vivo, many tumors appear persistent to oxidative stress. Thus, the finding that a diet rich in antioxidants or the elimination of ROS by antioxidant compounds prevents the development of certain types of cancer, provided the framework for further investigation of the tumorigenic actions of reactive oxygen species. This review presents current knowledge on the different roles of ROS in tumor development and progression.

Oxidative stress is defined as an imbalance between the production of free radicals and reactive metabolites, so-cal- 
led oxidants or ROS and their elimination through protective mechanisms, called antioxidants. This imbalance leads to the deterioration of biomolecules and important cells, with a potential impact on the whole organism [1]. ROS are products of a normal cellular metabolism and play vital roles in stimulating signaling pathways in plant and animal cells in response to changes in intra and extracellular environmental conditions [2]. Most ROS are generated in mitochondrial respiratory chain cells [3]. During endogenous metabolic reactions, aerobic cells produce ROS, such as superoxide anion $\left(\mathrm{O}^{2-}\right)$, hydrogen peroxide $\left(\mathrm{H}_{2} \mathrm{O}_{2}\right)$, hydroxyl radical $(\mathrm{OH} \bullet)$ and organic peroxides as normal products of the biological reduction of molecular oxygen [4]. The transfer of electrons to molecular oxygen takes place in the respiratory chain, and the electron transport chains located in the membranes of mitochondria $[5,6]$. Under hypoxic conditions, the mitochondrial respiratory chain also produces nitric oxide (NO), which can generate other reactive nitrogen species (RNS). RNS can further generate other reactive species, for example, reactive aldehydes-malondialdehyde (MDA) and 4-hydroxynonenal (4-HNE), by inducing excessive lipid peroxidation [7]. Proteins and lipids are also significant targets for oxidative attack, and modifying these molecules may increase the risk of mutagenesis [8].

The adverse biological activities of thiosemicarbazone (TSC) and Schiff base derivatives (SBD) have been widely studied in rats and other animal species, using different doses and routes of administration. However, there are few studies describing changes in vivo biochemical parameters that indicate the antioxidant system and oxidative stress in biological systems and morphological changes in tissues. In this study, rats were injected subcutaneously with thiosemicarbazone coordination compounds containing Schiff bases.

The aim of this study is to determine the effect of TSC coordination compounds on changes in the erythrocyte antioxidant system in rats that can be used to determine the efficacy of new indigenous preparations.

\section{Material and methods}

The research was approved by the Research Ethics Committee of Nicolae Testemitanu State University of Medicine and Pharmacy (protocol No 73 of 26.04.2017).

The study included new local copper coordination CC, thiosemicarbazide derivatives - TIA-160, CMT-67 and CMJ-33, synthesized at the State University of Moldova in the Laboratory of Advanced Materials in the Biopharmaceutical and Technical Field, under the supervision of Aurelian Gulea [9].

The action of local CC on the antioxidant system was evaluated in experiments on a group of 34 white male rats of the Wistar line weighing $180-230$ g, divided into 4 groups of 8-9 animals each. The first group - the control, consisted of 8 animals, maintained on a regular diet of vivarium and which were injected subcutaneously 3 times a week saline for 30 days.
Animals in experimental groups 2-4 were given subcutaneous CC 3 times a week for 30 days, over 30 days in the following sequence: group 2 - TIA-160 (0.1 $\mu \mathrm{M} / \mathrm{kg})$, lot 3 - CMT-67 (0.1 $\mu \mathrm{M} / \mathrm{kg})$ and lot 4 - CMJ-33 (0.1 $\mu \mathrm{M} / \mathrm{kg})$.

After 24 hours from the expiration of the experiments, blood was collected to evaluate the parameters of the antioxidant system. The erythrocyte mass, obtained after decanting the blood serum, was washed twice with saline.

Total antioxidant capacity (TAC) in erythrocytes was assessed using the methodology described by Pellegrini N., Ying M., Rice-Evans C., adapted to the multimodal rider with Synergy H1 Hydrid Reader plates (BioTek Instruments, USA) [10].

This method has been reported as a discoloration assay, applicable to both lipophilic and hydrophilic antioxidants. The preformed radical cation of 2,2'-azinobis- (3-ethylbenzothiazolin-6-sulfonic acid) $\left(\mathrm{ABTS}^{*}+\right)$ is generated by the oxidation of ABTS with potassium persulfate and is reduced in the presence of such hydrogen-donating antioxidants. The influences of both, the antioxidant concentration and the duration of the reaction on the inhibition of radical cationic absorption have been taken into account in determining the total antioxidant capacity.

Catalase Assay Kit and superoxide dismutase activity were performed using reagent kits obtained from local firms and performed according to the attached protocol.

The statistical evaluation of the obtained data was performed with the use of the computer program StatsDirect. The arithmetic mean \pm error of the mean $(\mathrm{M} \pm \mathrm{m})$ was calculated. The nonparametric statistical test " $U$ MannWhitney" and the significance threshold "p" $(p<0.05)$ were used to test the significant difference between the studied indices of the compared groups.

\section{Results}

The evaluation results of the antioxidant system indices: TAC with ABTS, CAT and SOD in erythrocytes when administering autochthonous CCs are presented in the statistics in table 1.

Table 1. Influence of autochthonous CCs, thiosemicarbazide derivatives, on the indices of the antioxidant system in erythrocytes TAC with ABTS

\begin{tabular}{|c|c|c|c|}
\hline $\begin{array}{l}\text { Groups of } \\
\text { study }\end{array}$ & $\begin{array}{c}\text { TAC with ABTS } \\
\mathrm{mM} / \mathrm{g} \cdot \mathrm{Hb}\end{array}$ & $\begin{array}{c}\text { Catalase, } \\
\mu \mathrm{M} / \text { s.g.Hb }\end{array}$ & $\begin{array}{l}\text { SOD, unit } \\
\text { conv } / \mathbf{g} \cdot \mathrm{Hb}\end{array}$ \\
\hline Contol group & $\begin{array}{c}38.53 \pm 1.35 \\
(100 \%)\end{array}$ & $\begin{array}{c}18.80 \pm 0.86 \\
(100 \%)\end{array}$ & $\begin{array}{c}6.72 \pm 0.42 \\
(100 \%)\end{array}$ \\
\hline $\begin{array}{c}\text { TIA-160 } \\
0.1 \mu \mathrm{M} / \mathrm{kg}\end{array}$ & $\begin{array}{c}36.08 \pm 0.84 \\
(94 \%)\end{array}$ & $\begin{array}{c}33.23 \pm 1.77^{* * * *} \\
(177 \%)\end{array}$ & $\begin{array}{c}7.12 \pm 0.47 \\
(106 \%)\end{array}$ \\
\hline $\begin{array}{c}\text { CMT-67 } \\
0.1 \mu \mathrm{M} / \mathrm{kg}\end{array}$ & $\begin{array}{c}29.09 \pm 0.68 * * * \\
(75 \%)\end{array}$ & $\begin{array}{c}23.69 \pm 3.23 * * \\
(126 \%)\end{array}$ & $\begin{array}{c}5.21 \pm 0.48 * * \\
(78 \%)\end{array}$ \\
\hline $\begin{array}{c}\text { CMG-33 } \\
0.1 \mu \mathrm{M} / \mathrm{kg}\end{array}$ & $\begin{array}{c}36.58 \pm 1.02 \\
(95 \%)\end{array}$ & $\begin{array}{c}27.66 \pm 1.81 * * * \\
(147 \%)\end{array}$ & $\begin{array}{c}6.39 \pm 0.52 \\
(95 \%)\end{array}$ \\
\hline
\end{tabular}

Note: ${ }^{*}$ - statistically significant difference with the control group $\left({ }^{*}-\mathrm{p}<0.05 ;^{* *}-\mathrm{p}<0.01 ;^{* * *}-\mathrm{p}<0.001\right)$. 
The animals exposed to the action of CC, thiosemicarbazone derivatives demonstrated a significant increase in erythrocyte catalase activity exceeding $1.3-1.8$ times the control values.

It was established that the compound TIA-160 $(0.1 \mu \mathrm{M} /$ $\mathrm{kg}$ ) showed a tendency to increase the activity of TAC and catalase by $39-51 \%$ compared to the control group, and SOD was found to be maintained within the control group.

The study reveals that SOD decreases statistically conclusively by $15 \%(\mathrm{p}<0.05)$ at the administration of the compound CMT-67 $(0.1 \mu \mathrm{M} / \mathrm{kg})$, and the total antioxidant activity decreases by $25 \%$, at the same time, the function of erythrocyte catalase in this case increases truthfully by $26 \%$. After the administration of the compound CMG-33 the CAT level increases by $47 \%$, the changes of the TAC content and of the SOD activity in this case proved to be inconclusive, these remaining within the limits of the values registered in the control group.

\section{Discussion}

In this study, was analyzed the activity of the antioxidant system in erythrocytes in laboratory animals subjected to the action of native CCs, thiosemicarbazide derivatives. TSC derivatives were in the focus of chemists and biologists due to their wide range of pharmacological effects. TSC derivatives showed highly effective antitumor properties in various types of tumors (leukemia, pancreatic cancer, breast, lung, cervical cancer, and prostate and bladder cancer). To obtain a better activity, different series of TSCs were developed by modifying the heteroaromatic system in their molecules. Thus, the antineoplastic activity became significant at the attachment of the side chain carbonyl at the a position to the ring nitrogen atom, while the attachment of the $\beta$ or $\gamma$ side chain to the $\mathrm{N}$ heterocyclic atom led to inactive antitumor agents [11].

Studies in recent years have provided increasing evidence of the fundamental importance of copper for the formation and functioning of several enzymes and proteins, such as $\mathrm{Cu} / \mathrm{Zn}$ superoxide dismutase, or cytochrome $\mathrm{C}$ oxidase, which are involved in superoxide oxygen neutralization processes, in tissue respiration, energy metabolism and DNA synthesis. The coordinating compounds of copper have been shown to be promising antitumor therapeutic agents that act through various mechanisms [12].

Acting to protect the body against certain harmful prooxidants, in this study was evaluated a complex system of enzymatic and non-enzymatic antioxidants, which included the determination of superoxide dismutase, catalase and total antioxidant capacity.

In this study were obtained significantly low levels of TAC and SOD activity, compared to the control group, at the administration of compound CMT-67, while the values of these indices under the influence of the other compounds studied did not have any changes with statistical significance.

The animals exposed to the action of CC, thiosemicar- bazone derivatives were found to have a significant increase in erythrocyte calalase activity that exceeded 1.3-1.8 times the control values, and the compound TIA-160 $(0.1 \mu \mathrm{M} / \mathrm{kg})$ shows the highest capacity to induce erythrocyte catalase expression. This indicates existence of an excessive synthesis of CAT after exposure to these compounds, a very important fact, established by us for the first time. On the other hand, this study showed that there are differences in the mechanisms of action of thiosemicarbazide derivatives. The significance and importance of the phenomenon of catalase induction by $\mathrm{CC}$, thiosemicarbazide derivatives emerge from the wide possibilities of application of these inducers in practice. Thus, these inducers could be used as promising remedies for the treatment and prevention of renal fibrosis induced by catalase deficiency [13], or the treatment of some forms of infertility, because catalase has been detected in the oocytes of mice, where it probably plays the role of protecting the genome from oxidative damage during meiotic maturation [14].

Taking into consideration the capacity of the thiosemicarbazide derivatives so easily penetrate the blood-brain barrier, along with their stability in the bloodstream, they may be used for the development of new effective methods for early diagnosis of severe infections of the brain, such as brain tumors and their metastasis, as well as visualization of $\mathrm{A} \beta$ plaques of Alzheimer's disease.

Similarly, catalase could be extremely useful for the development of effective therapy of brain and neurological disorders, proceeding from the fact that the catalase activity in the brain is extremely low compared to other tissues and organs, such as the liver and kidneys. The results of some studies reveal the importance of transient receptor potential (TRP) channels as a key component of the neurological pathway of $\mathrm{Ca}^{2+}$ ion entry in response to the harmful action of ROS. Explorers' data suggest that catalase may act effectively by suppressing the TRP channel activated by oxidative stress, showing protective effects on neuronal mitochondrial function and neuronal survival [15]. Catalase could also be extremely useful in the future for the development of effective therapies for neurodegenerative diseases, such as Alzheimer's and Parkinson's disease, as well as sensory pain, as the decrease in catalase activity by oxidative stress plays an important role in the etiopathogenesis of diseases mentioned above.

It has been established that catalase expression is also altered in cancer cells, which promotes cell proliferation by inducing genetic instability and activating oncogenes. Regulation of catalase expression is to be controlled primarily at transcriptional levels, although other mechanisms may be involved. In addition to transcription factors, such as Sp1 and NF-Y, transcription factors JunB and RARa are crucial regulators in breast cancer cells by recruiting proteins involved in transcriptional complexes and chromatin remodeling. Therefore, catalase may be an attractive therapeutic target in the context of cancer [16].

Under normal conditions the harmful effect of SOD is in balance with the antioxidant system. Antioxidant systems 
work together, not in isolation, because there are interactions between hydrophilic and lipophilic antioxidants.

SODs were the first antioxidant enzymes characterized [17]. Three different types of SOD are expressed in cells: cytosolic copper-zinc SOD (Cu-ZnSOD), mitochondrial MnSOD and extracellular SOD - SOD (EC-SOD), all of which are able to disperse two $\mathrm{O}_{2}-$ anions to $\mathrm{H}_{2} \mathrm{O}_{2}$ and molecular oxygen. Catalase is then responsible for detoxifying $\mathrm{H}_{2} \mathrm{O}_{2}$ into oxygen and water. These enzymes have specific distributions and functions. Their importance lies in protecting the tissues from the harmful action of various oxidants oxygen free radicals [18].

The function attributed to catalase is dismutation of $\mathrm{H}_{2} \mathrm{O}_{2}$ into oxygen and water, which is very important in the defense of cells against oxidative damage by $\mathrm{H}_{2} \mathrm{O}_{2}$. Hydrogen peroxide is not only toxic by its ability to form other ROS, such as the hydroxyl radical by the Fenton reaction, but $\mathrm{H}_{2} \mathrm{O}_{2}$ can act as a second messenger, being involved in multiple physiological and pathophysiological processes [19]. In addition, catalase can also act as a peroxidase, thus contributing to the metabolism of small substrates, such as methanol, ethanol, azide, hydroperoxides and, in the case of ethanol, is able to oxidize it to acetaldehyde, contributing to its metabolism in the liver. Thus, catalase may have additional roles, such as detoxification or activation of toxic and antitumor compounds.

In general, endogenous and physiological reactive oxygen species (ROS) are generated mainly in the process of oxidative reaction of the mitochondrial respiratory chain as by-products of normal cellular metabolism [20]. ROS have a comprehensive influence on cell physiology. Moderate amounts of ROS have positive effects, which include the destruction of invasive pathogens, wound healing and repair processes [21].

It is generally accepted that the cellular maintenance of redox homeostasis is controlled by a complex network of antioxidant enzymes (superoxide dismutases and glutathione peroxidases) whose expression is under the control of finetuning the Keap1-Nrf2 signaling pathway [22]. However, the molecular mechanisms that regulate the expression of catalase - the oldest known and discovered antioxidant enzyme - are independent of this pathway and are not fully elucidated, which would allow a new approach to modulate the antioxidant status in cancer cells in particular, in cases where its expression decreases [23]. Although the mechanisms that control catalase expression have been partially elucidated, low catalase expression in cancer cells remains an unanswered question. Under stress, the antioxidant enzyme catalase plays a major role in detoxifying $\mathrm{H}_{2} \mathrm{O}_{2}$.

As it is known, oxidative stress is an imbalance between the production of ROS and their elimination through multiple protection mechanisms, the activation of which can lead to chronic inflammation. Oxidative stress can activate a variety of transcription factors, which lead to the differential expression of genes involved in inflammatory pathways. Moreover, the levels of secondary messengers, such as cGMP, cAMP, protein kinases and calcium ions, transcrip- tion factors such as NF- $\kappa \mathrm{B}, \mathrm{AP}-1$, protooncogenes and some enzymes (iNOS, COX-2, proteases), cytokines and growth factors (IL-1 $\beta$, IL-6, TNF- $\alpha$, PDGF, VEGF, FGF-b, TGF-b, etc.) are known to be central in inflammatory processes. Thus, chronic inflammation is induced by biological, chemical and physical factors and is in turn associated with an increased risk of several types of human cancer and chronic diseases. The link between inflammation and cancer has been suggested by epidemiological and experimental data $[24,25]$ and confirmed by anti-inflammatory therapies that show efficacy in cancer prevention and treatment [26].

The fact that continuous irritation for long periods of time can lead to cancer has already described in the traditional Ayurvedic medical system (i.e. the science of long life), written 5000 years ago [27]. Whether this irritation is the same as what Rudolf Virchow called inflammation in the nineteenth century is uncertain. Rudolf Virchow first noted that inflammatory cells are present in tumors and that tumors occur in places of chronic inflammation [28]. This inflammation is considered a "secret killer" for diseases such as cancer. For example, inflammatory bowel disease, such as Crohn's disease and ulcerative colitis, is associated with an increased risk of colonic adenocarcinoma [29, 30], and chronic pancreatitis is associated with an increased rate of pancreatic cancer.

The exact mechanisms by which a wound healing process turns into cancer are the subject of intensive research [31], and possible mechanisms include induction of genomic instability, changes in epigenetic events and subsequent inappropriate gene expression, increased cell proliferation, resistance in apoptosis, aggressive tumor neo-vascularization, tumor-associated basement membrane invasion, and metastasis [32]. The way of oxidative stress modulates these different stages of inflammation-induced carcinogenesis is currently the subject of in-depth research. Sources of inflammation are widespread and include microbial and viral infections, exposure to allergens, radiation and toxic chemicals, autoimmune and chronic diseases, obesity, alcohol consumption, tobacco use, and a high-calorie diet [33].

In general, the longer the inflammation persists, the higher risk of cancer. There are two stages of inflammation, acute and chronic inflammation. Acute inflammation is an initial stage of inflammation (innate immunity), which is mediated by activating the immune system. This type of inflammation persists only for a short time and is usually beneficial to the host. If the inflammation lasts for a longer period of time, the second stage of inflammation or chronic inflammation sets in and may predispose the host to various chronic diseases, including cancer. During inflammation, mast cells and leukocytes are recruited at the site of injury, leading to a "respiratory explosion" due to increased oxygen uptake and thus increased release and accumulation of ROS at the site of injury [34].

Indigenous CCs are compounds with antioxidant properties, therefore they could manifest potential anti-inflammatory, antineoplastic, antiaging, antimicrobial and 
antiviral properties. Thus, they can find wide application in various pathological conditions and processes.

Further clarification and understanding of involved mechanisms will allow more precise definition of situations where local CC administration will prove beneficial. Such an investigation may also be useful for the development of new compounds with beneficial effects in the medication and prevention of multifactorial diseases.

\section{Conclusions}

The most informative biomarkers of antioxidant system functionality have been estimated and selected to assess the level of oxidative stress in erythrocytes on experimental exposure by administering native CCs to laboratory animals and which can be used to determine the efficacy of new native preparations.

The influence of tested CC on the indices of the antioxidant system is selective. This selectivity may be the basis of their strong selective antiproliferative and cytotoxic action on tumor cells, but not on healthy ones.

The elucidation of the molecular mechanisms underlying the action of CC broadens the theoretical knowledge about the biological properties of a number of chemical compounds and, at the same time, offers new possibilities to explore perspective objects in order to obtain new effective drug preparations.

\section{References}

1. Durackova Z. Some current insights into oxidative stress. Physiol Res. 2010;59(4):459-469. doi: 10.33549/physiolres.931844.

2. Jabs T. Reactive oxygen intermediates as mediators of programmed cell death in plants and animals. Biochem Pharmacol. 1999;57(3):231-245. doi: 10.1016/s0006-2952(98)00227-5.

3. Poyton RO, Ball KA, Castello PR. Mitochondrial generation of free radicals and hypoxic signaling. Trends Endocrinol Metab. 2009;20(7):332340. doi: 10.1016/j.tem.2009.04.001.

4. Fridovich I. The biology of oxygen radicals. Science. 1978;201:875-880. doi: $10.1126 /$ science. 210504.

5. Goossens V, De Vos K, Vercammen D, Steemans M, Vancompernolle K, Fiers W, Vandenabeele P, Grooten J. Redox regulation of TNF signaling. Biofactors. 1999;10(2-3):145-156. doi: 10.1002/biof.5520100210.

6. Goossens V, Grooten J, De Vos K, Fiers W. Direct evidence for tumor necrosis factor-induced mitochondrial reactive oxygen intermediates and their involvement in cytotoxicity. Proc Natl Acad Sci USA. 1995;92(18):8115-8119. doi: 10.1073/pnas.92.18.8115.

7. Hussain SP, Hofseth LJ, Harris CC. Radical causes of cancer. Nat Rev Cancer. 2003;3(4):276-285. doi: 10.1038/nrc1046.

8. Schraufstatter I, Hyslop PA, Jackson JH, Cochrane CG. Oxidant-induced DNA damage of target cells. J Clin Invest. 1988;82(3):1040-1050. doi: 10.1172/JCI113660.

9. Gulea A, Tsapkov V, Poirier D, et al. [Sulfanylcontaning copper (II) internal complexes with 2-[(2-hydroxifenilamino)-methyl]- fenol and 1-[(2-hy-droxifenilamino)-methyl]-naftalene-2-ol]. [Russ J General Chem]. 2010;40(3):212-218. Russian.

10. Gudumac V, Rîvneac V, Tagadiuc O, et al. Metode de cercetare a metabolismului hepatic: elaborare metodică [Methods of research of hepatic metabolism: methodical elaboration]. Chisinau: Tehnica-Info, 2012. 162 p. Romanian.

11. Shakya B, Yadav PN. Thiosemicarbazones as potent anticancer agents and their modes of action. Mini Rev Med Chem. 2020;20(8):638-661. doi: 10.2174/1389557519666191029130310.
12. Krasnovskaya O, Naumov A, Guk D, et al. Copper coordination compounds as biologically active agents. Int J Mol Sci. 2020 Jun;21(11):3965. doi: 10.3390/ijms 21113965 .

13. Kobayashi M, Sugiyama H, Wang DH, et al. Catalase deficiency renders remnant kidneys more susceptible to oxidant tissue injury and renal fibrosis in mice. Kidney Int. 2005;68(3):1018-1031. doi: 10.1111/j.15231755.2005.00494.x.

14. Park YS, You SY, Cho S, Jeon HJ, Lee S, Cho DH, Kim JS, Oh JS. Eccentric localization of catalase to protect chromosomes from oxidative damages during meiotic maturation in mouse oocytes. Histochem Cell Biol. 2016;146(3):281-288. doi: 10.1007/s00418-016-1446-3.

15. Nazıroğlu M. Molecular role of catalase on oxidative stress-induced $\mathrm{Ca}^{2+}$ signaling and TRP cation channel activation in nervous system. J Recept Signal Transduct Res. 2012;32(3):134-141. doi: 10.3109/10799893.2012.672994.

16 Glorieux C, Calderon PB. Catalase, a remarkable enzyme: targeting the oldest antioxidant enzyme to find a new cancer treatment approach. Biol Chem. 2017;398(10):1095-1108. https://doi.org/10.1515/hsz-2017-0131.

17. Schetter AJ, Heegaard NH, Harris CC. Inflammation and cancer: interweaving microRNA, free radical, cytokine and p53 pathways. Carcinogenesis. 2010;31(1):37-49. doi: 10.1093/carcin/bgp272.

18. Sies H. Hydrogen peroxide as a central redox signaling molecule in physiological oxidative stress: oxidative eustress. Redox Biol. 2017;11:613-619. doi: 10.1016/j.redox.2016.12.035.

19. He L, He T, Farrar S, Ji L, Liu T, Ma X. Antioxidants Maintain Cellular Redox Homeostasis by Elimination of Reactive Oxygen Species. Cell Physiol Biochem. 2017;44(2):532-553. doi: 10.1159 / 000485089.

20. Bhattacharyya A, Chattopadhyay R, Mitra S, Crowe SE. Oxidative stress: an essential factor in the pathogenesis of gastrointestinal mucosal diseases. Physiol Rev. 2014;94(2):329-354. doi: 10.1152/physrev.00040.2012.

21. Menegon S, Columbano A, Giordano S. The dual roles of Nrf2 in cancer. Trends Mol Med. 2016;22(7):578-593. doi: 10.1016/j.molmed.2016.05.002.

22. Cullen JJ, Mitros FA, Oberley LW. Expression of antioxidant enzymes in diseases of the human pancreas: another link between chronic pancreatitis and pancreatic cancer. Pancreas. 2003;26(1):23-27. doi: 10.1097/00006676-200301000-00005.

23. Bartsch H, Nair J. Chronic inflammation and oxidative stress in the genesis and perpetuation of cancer: role of lipid peroxidation, DNA damage, and repair. Langenbecks Arch Surg. 2006;391(5):499-510. doi: 10.1007/s00423-006-0073-1.

24. Grivennikov SI, Greten FR, Karin M. Immunity, inflammation, and cancer. Cell. 2010;140(6):883-899. doi: 10.1016/j.cell.2010.01.025.

25. Grivennikov SI, Karin M. Inflammation and oncogenesis: a vicious connection. Curr Opin Genet Dev. 2010;20(1):65-71. doi: 10.1016/j. gde.2009.11.004.

26. Garodia P, Ichikawa H, Malani N, Sethi G, Aggarwal BB. From ancient medicine to modern medicine: ayurvedic concepts of health and their role in inflammation and cancer. J Soc Integr Oncol. 2007;5(1):25-37. doi: 10.2310/7200.2006.029.

27. Aggarwal BB, Gehlot P. Inflammation and cancer: how friendly is the relationship for cancer patients? Curr Opin Pharmacol. 2009;9(4):351369. doi: 10.1016/j.coph.2009.06.020.

28. Ekbom A, Helmick C, Zack M, Adami HO. Increased risk of largebowel cancer in Crohn's disease with colonic involvement. Lancet. 1990;336:357-359. doi: 10.1016/0140-6736(90)91889-i.

29. Gillen CD, Walmsley RS, Prior P, Andrews HA, Allan RN. Ulcerative colitis and Crohn's disease: a comparison of the colorectal cancer risk in extensive colitis. Gut. 1994;35(11):1590-1592. doi: 10.1136/ gut.35.11.1590.

30. Ekbom A, McLaughlin JK, Nyren O. Pancreatitis and the risk of pancreatic cancer. N Engl J Med. 1993;329(20):1502-1503. doi: 10.1056/ NEJM199311113292016.

31. Coussens LM, Werb Z. Inflammation and cancer. Nature. 2002;420:860867. doi: 10.1038/nature01322.

32. Kundu JK, Surh YJ. Inflammation: gearing the journey to cancer. Mutat Res. 2008;659(1-2):15-30. doi: 10.1016/j.mrrev.2008.03.002. 33. 
Aggarwal BB, Vijayalekshmi RV, Sung B. Targeting inflammatory pathways for prevention and therapy of cancer: short-term friend, long-term foe. Clin Cancer Res. 2009;15(2):425-430. doi: 10.1158/1078-0432.CCR08-0149.
34. Lin WW, Karin M. A cytokine-mediated link between innate immunity, inflammation, and cancer. J Clin Invest. 2007;117(5):1175-1183. doi: $10.1172 /$ JCI31537.

\author{
Authors' ORCID iDs and academic degrees \\ Valeriana Pantea, MD, PhD Applicant - https://orcid.org/0000-0002-8835-6612 \\ Marina Gamaniuc, MD, PhD Applicant - https://orcid.org/0000-0002-7662-0973 \\ Veaceslav Popa, MD, PhD Applicant - https://orcid.org/0000-0003-3139-2652
}

\title{
Authors' contribution
}

VP designed the research, reviewed statistics and interpreted the data, revised the data and drafted the manuscript; MG conceptualized the project and designed the research, drafted the manuscript; VP conducted/ perfomed the laboratory work, revised the manuscript critically. All the authors revised and approved the final version of the manuscript.

\section{Funding}

This study was supported by Nicolae Testemitanu State University of Medicine and Pharmacy. The trial was the authors' initiative; the authors are independent and take responsibility for the integrity of the data and accuracy of the data analysis.

\section{Ethics approval and consent to participate}

The research protocol was approved by the Research Ethics Committee of Nicolae Testemitanu State University of Medicine and Pharmacy (protocol No 73 of 26.04.2017) and the tests have been done according to the contemporary principals in biological standardization of experiences and Declaration of Helsinki with further amendments (Somerset West Amendment, 1996).

\section{Conflict of Interests}

No competing interests were disclosed. 